\title{
Lifespan Normative Data on Rates of Brain Volume Changes
}

M. Battaglini ${ }^{1}$, G. Gentile ${ }^{1}$, L. Luchetti ${ }^{1}$, A. Giorgio ${ }^{1}$, H. Vrenken ${ }^{2}$, F. Barkhof ${ }^{2-3-4}$, K.S. Cover ${ }^{2,5}$, R. Bakshi $^{6}$, R. Chu ${ }^{6}$, M.P. Sormani ${ }^{7}$, C. Enzinger ${ }^{8-9}$, S. Ropele ${ }^{8}$, O. Ciccarelli ${ }^{10-4}$, C. WheelerKingshott $^{10-11-12}$, M. Yiannakas ${ }^{10}$, M. Filippi ${ }^{13}$, M.A. Rocca ${ }^{13}$, P. Preziosa ${ }^{13}$, A. Gallo ${ }^{14}$, A. Bisecco $^{14}$, J. Palace ${ }^{15}$, Y. Kong ${ }^{16-17}$, D. Horakova ${ }^{18}$, M. Vaneckova ${ }^{19}$, C. Gasperini ${ }^{20}$, S. Ruggeri ${ }^{20}$, N. De Stefano ${ }^{1}$ on behalf of the MAGNIMS Study Group

${ }^{1}$ Department of Medicine, Surgery and Neuroscience, University of Siena, Siena, Italy

${ }^{2}$ Department of Radiology and Nuclear Medicine, MS Center Amsterdam, Amsterdam, The Netherlands

${ }^{3}$ Institutes of Neurology and Healthcare Engineering, UCL London, UK

${ }^{4}$ National Institute for Health Research (NIHR), University College London Hospitals (UCLH)

Biomedical Research Centre (BRC), London, UK

${ }^{5}$ Department of Physics and Medical Technology, VU University medical Center, Amsterdam, The Netherlands

${ }^{6}$ Laboratory for Neuroimaging Research, Ann Romney Center for Neurologic Diseases, Brigham and Women's Hospital, Harvard Medical School, Boston, MA, USA

${ }^{7}$ Department of Health Sciences (DISSAL), University of Genoa, Genoa, Italy

${ }^{8}$ Department of Neurology, Medical University of Graz, Graz, Austria

${ }^{9}$ Division of Neuroradiology, Vascular \& Interventional Radiology, Department of Radiology, Medical University of Graz, Graz, Austria

${ }^{10}$ Queen Square Multiple Sclerosis Centre, UCL Institute of Neurology, University College, London, UK

${ }^{11}$ Brain MRI 3T Research Center, C. Mondino National Neurological Institute, Pavia, Italy

${ }^{12}$ Department of Brain and Behavioural Sciences, University of Pavia, Pavia, Italy

${ }^{13}$ Neuroimaging Research Unit, Institute of Experimental Neurology, Division of Neuroscience, San Raffaele Scientific Institute, Vita-Salute San Raffaele University, Milan, Italy

${ }^{14}$ Department of Medical, Surgical, Neurological, Metabolic and Aging Sciences, University of Campania Luigi Vanvitelli, Naples, Italy

${ }^{15}$ Nuffield Department of Clinical Neurosciences, Oxford University Hospitals NHS Trust, University of Oxford, Oxford, UK

${ }^{16}$ CAS Key Laboratory of Behavioral Science, Institute of Psychology, Beijing 100101, China

${ }^{17}$ Department of Psychology, University of Chinese Academy of Sciences, Beijing 100049, China

${ }^{18}$ Department of Neurology and Center of Clinical Neuroscience, First Faculty of Medicine, Charles University and General University Hospital in Prague, Czech Republic

${ }^{19}$ Department of Radiodiagnostics, First Faculty of Medicine Charles University and General University Hospital in Prague, Czech Republic

${ }^{20}$ Department of Neurosciences S Camillo Forlanini Hospital, Rome, Italy

Running title: Normal values of brain volume changes 
Corresponding Author:

Nicola De Stefano, MD PhD

Department of Medicine, Surgery and Neuroscience

University of Siena

Viale Bracci 2

53100 Siena, Italy

Tel: +39-0577-235808

Fax: +39-0577-233411

E-mail: destefano@unisi.it

Keywords: normative data, atrophy, aging, MRI, brain volume 


\begin{abstract}
We provide here normative values of yearly percentage brain volume change (PBVC/y) as obtained with SIENA, a widely used open-source software, developing a PBVC/y calculator for assessing the deviation from the expected PBVC/y in patients with neurological disorders. We assessed multicenter (34 centers, 11 MRI protocols) MRI data of 720 healthy participants covering the whole adult lifespan (16-90 years). Data of 421 participants with a follow-up > 6 months were used to obtain the normative values for PBVC/y and data of 392 participants with a follow-up <1 month were selected to assess the intra-subject variability of the BV measurement. A mixed model evaluated PBVC/y dependence on age, sex and MRI parameters (scan vendor and magnetic field strength). PBVC/y was associated to age $(\mathrm{p}<0.001)$, with 60-70 year-old participants showing twice more volume decrease than participants aged 30-40 years. PBVC/y was also associated to magnetic field strength, with higher decreases when measured by $1.5 \mathrm{~T}$ than $3 \mathrm{~T}$ scanners $(\mathrm{p}<0.001)$. The variability of PBVC/y normative percentiles (NP) was narrower as the inter-scan interval was longer (e.g., $80^{\text {th }} \mathrm{NP}$ was $50 \%$ smaller for participants with 2-year than with 1-year follow-up). The use of these normative data, eased by the freely available calculator, might help in better discriminating pathological from physiological conditions in many clinical settings.
\end{abstract}




\section{Introduction}

Neurodegenerative disorders are characterized by clinically relevant, irreversible brain tissue loss (i.e., atrophy) that begins at early stages and progresses relentlessly throughout the course of the disease (Frisoni et al., 2010; Rocca et al., 2017). Measures of brain volume changes as derived by magnetic resonance imaging (MRI) have been used in these disorders as index of neurodegeneration (Hotter et al., 2009; De Stefano et al., 2014; van Maurik et al., 2017) and, since halting neurodegeneration and promoting neuroprotection are the main targets of current therapeutic strategies in neurodegenerative diseases, these measures have been extensively applied in the context of both research studies and clinical trials (Giorgio and De Stefano, 2013).

Over the last two decades, substantial effort has been spent in improving MRI-derived methods of brain volume (BV) measurement. This has led to significant technical advancements, with several automated software tools that have shown ability in measuring BV loss with sensitivity, reproducibility, accuracy and precision (Vrenken et al., 2013). However, due to a number of constraints, at present the assessment of $\mathrm{BV}$ loss is not routinely performed in clinical practice (Rocca et al., 2017; Sastre-Garriga et al., 2017, De Stefano et al., 2017). In this context, the lack of normative data does not allow the quantification of the variability due to different acquisition protocols and scanner types in BV measurement and therefore the use of these measurements becomes particularly challenging in the clinical setting. Indeed, normative data of MRI-derived measures of BV are important as they i) may clarify the impact of important technical issues on the $\mathrm{BV}$ measures and ii) may be relevant in assessing deviations from normal behavior, as expected for age, sex and MRI acquisition settings, both at group and individual levels.

Some studies have assessed MRI-derived BV changes in healthy individuals (Liu et al., 2003; Fotenos et al., 2005; Driscoll et al., 2009; Marcus et al., 2010; Takao et al., 2012; Opfer et al., 2017). Most of them, however, have investigated BV changes on populations with limited age span (i.e., older adult) (Liu et al., 2003; Fotenos et al., 2005) and, even when performed over a broader range of adulthood, they had small sample sizes or were center-specific (i.e., using single MR 
scanner and acquisition protocol) (Takao et al., 2012; De Stefano et al., 2016; Opfer et al., 2017). Overall, these studies seem to suggest that the rate of atrophy increases with age and that there are not significant sex-related differences in atrophy rates (Liu et al., 2003; Takao et al., 2012). By and large, the most comprehensive description of the rate of BV loss occurring in healthy individuals over the lifespan probably comes from a recent meta-analysis of 56 different longitudinal studies (Hedman et al., 2012). However, due to its design (i.e., analysis of aggregate statistics from each longitudinal study), this study was also unable to provide answers on the role of different MRI acquisition protocols and settings, thus limiting the clinical utility of the resulting rates of BV changes. A large longitudinal study assessing individual data processed in a standard fashion is therefore still lacking.

Against this background, the present study aimed to assess, in a relatively large population of healthy individuals covering the whole adult lifespan, longitudinal BV changes from MRI data acquired in multiple centers. The size of the analysed population allowed addressing issues such the relevance of sex and age on BV changes with higher statistical power than in the past. The primary goal was to provide normative values of yearly percentage brain volume change (PBVC/y) as obtained with SIENA (Structural Imaging Evaluation, using Normalization, of Atrophy (Smith et al., 2002)), which is a widely used open-source software. The presence of a large, multi-center scan-rescan MRI dataset of healthy individuals with variable follow-up length allowed the MRrelated sources of variability (e.g., different vendors, magnetic field strengths, acquisition parameters and within-patient $\mathrm{BV}$ fluctuations) to be estimated and these were all taken into account in the statistical model that provides normative values of the rates of BV change.

\section{Materials and Methods}

\section{Participants}

This is a multicenter, retrospective study based on the analysis of longitudinal MR datasets of healthy individuals. A total of 3077 anonymized conventional 3D T1-weighted (T1-W) images of 
720 healthy individuals were collected from (Table 1) i) data of eight European imaging centers of the MAGNIMS (Magnetic Resonance Imaging in MS; www.magnims.eu) network Laboratory for Neuroimaging Research, Harvard Medical School, Boston, MA, USA, iii) data of the Department of Radiodiagnostics, Charles University, Prague, Czech Republic, and iv) a selected dataset of the ADNI (Alzheimer's Disease Neuroimaging Initiative; www.loni.usc.edu/ADNI) network, mainly older participants scanned on 1.5 Tesla (T) GE scanners. Details on demographics and MRI characteristics of the healthy population in each participating center are reported in Table 1. Out of the 720 healthy individuals, a subset of 421 participants with a follow-up $>6$ months was used to obtain the normative values for PBVC/y (Table 2) and a subset of 392 participants with a follow-up $<1$ month was selected to assess the intra-subject variability of the BV measurement using the SIENA software in different acquisition settings (Table 3). At each center, participants were included if they had no history of neurological or psychiatric disorders and if all T1-W images were acquired with the same procedure/sequence and scanner. For each dataset, MRI scans were acquired after consent obtained from each participant in each center. The final study protocol was reviewed and approved by the European MAGNIMS collaboration for the analysis of pseudoanonymized scans.

\section{MRI acquisition}

Data are summarized in Table 1 . MRI datasets came from 10 academic centers $(n=622)$ and the ADNI database ( $\mathrm{n}=98$ from 24 sites with identical acquisition protocol) and were acquired on scanners at both 1.5 and $3 \mathrm{~T}$ from the three leading vendors (i.e., Siemens Healthcare, Philips Medical Systems and GE Healthcare). There were no limits on the between-scan interval and on the number of scans for each participant. For each participant, data were acquired with the same scanner and same acquisition protocol for the whole follow-up period. None of the scanners were changed or underwent major upgrades during the study period. Each dataset was sent to the 
Quantitative Neuroimaging Laboratory of the University of Siena for quality control and centralized analysis.

\section{MRI data analysis}

Image pre-processing

An initial quality control was visually performed by expert neuroimagers (MB and $A G)$ in order to exclude images with artefacts. To avoid errors during the brain extraction procedure, large nonbrain sections (i.e., neck) were removed by using the standard_space_roi tool, part of FSL (FMRIB Software Library, www.fmrib.ox.ac.uk/fsl/;(Jenkinson et al., 2012)). Bias field correction on T1-W images was performed with the nonparametric nonuniform intensity normalization (N4) algorithm (Nakamura et al., 2013), part of ANTs v2.10 (Advanced Normalization Tools, http://stnava.github.io/ANTs/)(Tustison et al., 2010). Images that failed the intensity inhomogeneity correction were excluded from analysis.

SIENA analysis

Global brain volume changes over time were quantified using the SIENA method (Smith et al., 2002), also part of FSL (version 5.0.10). This registration-based method uses images from two time-points to assess BV changes by directly estimating the local shifts in brain edges across the entire brain and then converting the edge displacement into a global estimate of PBVC between the two time-points. While using SIENA, a procedure of brain extraction able to improve the removal of non-brain tissues was implemented for a more accurate BV estimation. The aim was to ensure that a consistent segmentation across time-points of the intracranial region, which is the starting point of the SIENA analysis, was considered in the analysis of multiple MRI scans. Thus, we obtained within-subject brain mask as follows:

i) since previous studies (Popescu et al., 2012; Cover et al., 2014) showed that the performance of the FSL brain extraction tool (BET, (Smith, 2002)) largely improves when using the bias field 
correction option "-B" with small values (i.e., 0.1 or 0.2 ) of the fractional intensity threshold option “-f”, for each time-point image of the participant, 5 different brain masks were obtained by using the "-B" option and 5 different values of the "-f" option (from 0.05 to 0.25 , increasing by 0.05 ). Among those masks, the one ensuring the best separation of brain from non-brain tissue was selected, based on visual inspection, and its options were used for each participant's time-point.

ii) the brain masks for each participant were aligned in the participant space, by using the results of the Anatomical-Average tool in FSL, which was run using the T1-weighted images.

iii) a single preliminary subject-brain mask was created by merging all the aligned time-point masks of that participant and retaining voxels that were segmented as brain in more than $50 \%$ of the masks.

iv) if necessary, as final refinement, a manual editing of the preliminary subject-brain mask was performed and thus the within-subject brain mask was obtained.

v) finally, the within-subject mask was transformed separately using trilinear interpolation into the 3D T1-W space of each time-point and subsequently used to perform the final brain extraction.

Then, SIENA was run on each pair of images and data outputs were visually checked by expert neuroimagers (MB and $\mathrm{AG})$ for a final quality assessment.

\section{Statistical analysis}

\section{Intra-subject variability}

Out of the whole dataset of 720 healthy individuals, a subset of 392 participants with a follow-up $<1$ month was selected to assess the intra-subject variability of the BV measurement using the SIENA software in different acquisition settings. Given the interval between the scans in each participant, the intra-subject variability can be considered to include both physiological fluctuations of BV and the measurement error. The intra-subject variability was quantified as the absolute error of PBVC. 
Generalized mixed linear models using the PBVC between two scans as dependent variable, magnetic field strength, vendor and their interaction as fixed factors and adding center as random variable, were performed to explore the PBVC intra-subject variability in different acquisition settings. The analysis was run using the R software (https://www.r-project.org/).

\section{Normative data}

Out of the 720 healthy individuals, a subset of 421 participants with a follow-up $>6$ months was used to obtain the normative values for PBVC/y. An arctangent (atan) transform was used to make the variance of PBVC/y independent of its mean value. Thus, analysis was performed by using the $\operatorname{atan}(\mathrm{PBVC} / \mathrm{y})$ as dependent variable.

To select the variables independently associated to PBVC/y, stepwise mixed models with random intercept were run, using mid-point age between the two scans as covariate and sex, magnetic field strength, presence of distortion correction and vendor as fixed factors. The final model included all the factors significantly $(\mathrm{p}<0.05)$ associated to PBVC/y at multivariate analysis. The standard deviation of the residuals was used to create the normative range.

The mixed-effects model with participant as random effects is known to deal with the presence of multiple scan pairs for a given participant taking into account also the auto-correlation structure of measures repeated over time on the same participant. This modeling approach allows obtaining i) the components of the variance of PBVC/y as the inter-subject variance $\left(\sigma_{\text {Inter subj } j}^{2}\right)$, that is linearly dependent on the length of follow-up, and ii) the intra-subject variance $\left(\sigma_{\text {Intra subj }}^{2}\right)$, which is the variation due to the sum of the volume changes related to random physiological conditions at the scan time and the error of the software, and that is independent on the length of follow-up. Thus, naming $f u$ the length of follow-up, $\sigma^{2}$ Inter subj-1y the inter-subject variance at 1 year of follow-up and $\sigma^{2}$ Intra subj the fixed intra-subject variance, the total variance estimated by the final mixed model is the sum of these variance components according to the following formula: 


$$
s d_{f u}=\sqrt{\sigma_{\text {Inter subj-1y }}^{2}+\frac{\left(\sigma_{\text {Intra } s_{u b j}}^{2}\right)}{f u^{2}}}
$$

The normative percentiles of PBVC/y were calculated using the formula:

$C I_{f u}^{\text {year }}=\tan \left(Y_{\text {predicted }}^{f u} \pm X X^{*} s d_{f u}\right)$

where $Y_{\text {predicted }}^{f u}$ is the PBVC/y as obtained by the regression model, XX was 0.84 for the $80^{\text {th }}$ and 1.64 for the $95^{\text {th }}$ percentile respectively, as obtained by the one-sided $\mathrm{Z}$ distribution. Data were converted to the original scale by applying the inverse of the arctangent transform.

\section{Results}

\section{Intra-subject variability of SIENA measurements}

Data used for assessing the intra-subject variability are summarized in Table 2. Out of the 392 healthy individuals with MRI datasets of $<1$ month follow-up, $12(3.07 \%)$ were excluded after quality control and a total of 536 SIENA analyses were run, as a number of participants had more than 1 pair of MRI scans.

The mean of the intra-subject variability, defined as the absolute error of PBVC, which includes the measurement error and short-term biological fluctuations (Opfer et al., 2018), was 0.25\% (Table 2). Small and non significant differences in intra-subject variability were found between scans acquired at $1.5 \mathrm{~T}$ and those acquired at $3 \mathrm{~T}$ (mean: $0.22 \%$ at $1.5 \mathrm{~T}, 0.25 \%$ at $3 \mathrm{~T}$ ); data acquired with Philips scanners had slightly higher intra-subject variability than those acquired with Siemens and GE (mean: $0.28 \%$ for Philips, $0.17 \%$ for Siemens, $0.23 \%$ for GE).

\section{Prediction of PBVC/y}

Data used for assessing the normative BV rates are summarized in Table 3. Out of the 421 healthy individuals with follow-up MRI > 6 months, 37 (8.78\%) were excluded after quality control and a 
total of 789 SIENA analyses were run (Figure 1), as a number of participants had more than 1 pair of MRI scans. The age of the group ranged from 16 to 90 years (mean \pm SD: $45.7 \pm 18.4$ years) and $53.6 \%$ were female.

The model with the best fit for atan(PBVC/y), as assessed by the stepwise mixed model analysis, selected age and magnetic field strength as independent significant predictors $\left(R^{2}=0.18 ; p<0.001\right)$. The other factors such as sex, scanner vendor and presence of distortion correction were discarded by the model.

\section{Effect of magnetic field strength}

PBVC/y measured on $3 \mathrm{~T}$ scanners showed significantly smaller atrophy rate $(0.127 \%, \mathrm{p}<0.001)$ than those on $1.5 \mathrm{~T}$.

\section{Effect of age}

The mean PBVC/y showed age-related increase over the adult lifespan. PBVC/y per age period and magnetic field strength are reported in Table 4 and Table 5, ranging from $-0.13 \%$ at $1.5 \mathrm{~T}$ and $0.02 \%$ at $3 \mathrm{~T}$ at the age of 20 years to $-0.54 \%$ at $1.5 \mathrm{~T}$ and $-0.4 \%$ at $3 \mathrm{~T}$ at the age of 80 years.

\section{Normative percentiles}

The intra-subject variability estimated by the mixed model showed a standard deviation of $0.29 \%$, which was close to the value estimated by using the scan pairs with follow-up $<1$ month. The intersubject variability of PBVC/y was estimated as a standard deviation of $0.16 \%$.

As expected, the normative percentiles of PBVC/y showed an age-related increase over the adult lifespan. There were also significant differences when normative percentiles of PBVC/y were measured at 1.5 or $3 \mathrm{~T}$ (Figure 2). Further, because the contribution of the intra-subject variability to the whole variance is divided by the follow-up length (see Formula 1), the variability of PBVC/y was narrower as the inter-scan interval was longer. For example, at $1.5 \mathrm{~T}$ the $80^{\text {th }}$ normative 
percentile of PBVC/y ranged from $-0.48 \%$ at the age of 20 years to $-0.92 \%$ at the age of 80 years when the PBVC was estimated over 1 year (see Table 4). Over 2-year follow-up, the $80^{\text {th }}$ normative percentiles of PBVC/y values at $1.5 \mathrm{~T}$ ranged from $-0.37 \%$ at the age of 20 years to $-0.76 \%$ at the age of 80 years. In the same conditions, at $3 \mathrm{~T}$ the $80^{\text {th }}$ normative percentiles of PBVC/y was $-0.34 \%$ at the age of 20 years and $-0.73 \%$ at the age of 80 years for 1 -year follow-up, and $-0.24 \%$ at the age of 20 years and $-0.59 \%$ at the age of 80 years for 2-year follow-up (Table 5).

On the MAGNIMS website (www.magnims.eu), a calculator will be made available to automatically calculate the expected PBVC/y for a given individual according to the relevant variables such as age, magnetic field strength and inter-scan interval as inputs.

\section{Discussion}

The present work provided normative values of annualized total BV change (i.e., PBVC/y) as assessed by SIENA, an open-source software that is widely used for the assessment of BV changes on clinical MR scans (Mak et al., 2015; Sastre-Garriga et al., 2015; Guevara et al., 2017). Great effort has been devoted in pooling MRI data from a relatively large population of healthy individuals that were acquired from 34 different scanners with 11 different acquisition protocols. Thus, the study population should be representative of the whole adulthood (with age ranging from 16 to 90 years) and covers most of the real-world acquisition protocols, with MRI examinations performed with the three most used MR scanner vendors (i.e., General Electrics, Philips, Siemens) and clinical magnetic field strengths (i.e., 1.5T and 3T). Interestingly, results clearly showed that, among all parameters, age and magnetic field strength were the significant predictors of PBVC/y.

Data analysis showed an acceleration of BV loss during aging, with values of PBVC/y that doubled in individuals aged 60-70 years in comparison to those aged 30-40 years, independently of the MRI setting. This finding is expected (Liu et al., 2003; Takao et al., 2012) and was hypothesized as due to different temporal patterns of grey matter (GM) and white matter (WM) volume change, with the former linearly decreasing after adolescence and the latter being stable or slightly increasing until 
45 years and decreasing thereafter (Hedman et al., 2012). This hypothesis could not be tested here because SIENA does not allow separate longitudinal analyses of GM and WM volume changes. However, we plan to pursue this goal in the near future by using some of the recently proposed methods (Reuter and Fischl, 2011; Ashburner and Ridgway, 2013; Battaglini et al., 2018). In general, however, it is worth stressing that the increased range of normality with aging might also reflect a larger variability in the rate of BV change in older adults (Enzinger et al., 2005). Previous studies (De Stefano et al., 2017) have shown that presence/absence of specific habits (i.e., smoking, alcohol consumptions, etc.) and/or comorbidities (i.e., hypertension, diabetes, obesity, etc.) do have an impact on the rate of BV change, particularly with increasing age, and this might have had a significant role in the increased variability (i.e., increased PBVC/y) observed in our older participants (Resnick et al., 2003). Unfortunately, the retrospective design of the study did not allow the retrieval of this information.

As mentioned before, the multicenter data analysed in the present study were acquired with different protocols $(n=11)$, on a relatively large number of MR scanners $(n=34)$ by the three most popular vendors and with the two magnetic field strengths currently used in the clinic setting (i.e., 1.5T and 3T). Interestingly, our results showed that different MR protocols and scanner types did not add significant prediction to the rate of BV changes. However, our findings did show difference in BV rates on data acquired on scanners with different magnetic field strengths. This held true also in subgroup analyses of participants grouped for age, with the exclusion of those $>65$ of age as they did not have scan acquisition at $3 \mathrm{~T}$ (see Fig 1, data not shown). Since the two cohorts (1.5 T and 3T) included different individuals and differences could therefore be caused by cohort effects, we increased confidence in this finding by performing a post-hoc analysis on 41 healthy individuals from the ADNI dataset who had 1-year follow-up data acquired at both 1.5T and 3T. Lower BV changes were found on data acquired at 3T $(-0.51 \% \pm 0.8)$ with respect to those acquired at $1.5 \mathrm{~T}(-$ $0.68 \% \pm 0.9, \mathrm{p}=0.04$, data not shown). Since $3 \mathrm{~T}$ images may have a better contrast at the GM/cerebrospinal fluid (CSF) interface than 1.5T images (Kruggel et al., 2010; Chu et al., 2016; 
Chu et al., 2017), this could have a great impact on the SIENA analysis, which is essentially based on the quantification of the shift at GM/CSF interface between two time-points. However, other causes such as different coil design (including parallel imaging), pulse sequence choice, k-space sampling schemes and gradient strength might also explain the differences in BV changes between data acquired at $1.5 \mathrm{~T}$ and $3 \mathrm{~T}$, a finding that deserves further investigation.

When assessing MRI-derived BV changes in a population, the total variance comes from differences between individuals (inter-subject variability) and from the random fluctuation of BV in each individual (intra-subject variability). Due to the characteristics of our dataset, we were able to measure here both types of variability. The intra-subject variability, which includes the measurement error and short-term biological fluctuations, in our study was very close to the withinpatient fluctuations recently described by Opfer and co-workers (Opfer et al., 2018). In addition, in line with recent studies (Biberacher et al., 2016; Opfer et al., 2018), we found here a decreased intra-subject variability with longer follow-up (i.e., 2 years vs 1 year). This has practical implications as, in providing normative percentiles of $\mathrm{PBVC} / \mathrm{y}$, the cut-off values are definitely influenced by measurement errors and short-term biological fluctuations, so that the interval between the expected value and the $95^{\text {th }}$ normative percentiles of PBVC/y was $40 \%$ smaller if obtained from MRI examinations at 2-year than at 1-year follow-up. In our dataset, for example, if the range of normality is defined as the positive difference between the expected value and the $95^{\text {th }}$ normative percentiles of PBVC/y, this was $40 \%$ more accurate if obtained from MRI examinations at 2-year than at 1-year follow-up. This suggests the need for a "dynamic" approach when using pathological cut-off values in clinical practice, in which the rate of BV change for a single patient should be assessed using a model (e.g., a calculator) that incorporates the various important factors, such as age, magnetic field strength and follow-up time. It is worth stressing that the proposed normative data have a larger error than those proposed in previous studies (De Stefano et al., 2016; Opfer et al., 2017). The above-mentioned relevance of the follow-up length as well as the use in the 
present study of a large, multicenter dataset with multiple acquisition protocols and settings can represent plausible explanations for this apparent discrepancy.

Overall, results reported in the present study showed that MRI-derived rates of BV changes are dependent on the age, magnetic field strength and follow-up length. All these parameters will be therefore included in a freely available calculator (www.magnims.eu), which is meant to automatically provide the normative range of PBVC/y for a given individual and, thus, the potential deviation from healthy conditions. It should be stressed here that the calculator output should not be used per se, but needs to be contextualised with all other individual information by the referring doctor. While this tool does not overcome all constraints hampering the use of BV loss assessments in clinical practice (Rocca et al., 2017; Sastre-Garriga et al., 2017, De Stefano et al., 2017) and could certainly be improved in the future by adding further data and more refined assessments, it presently includes carefully assessed normative data of the rate of BV change that are representative of the whole adult lifespan and were acquired with the most frequent settings used in clinic. This should represent an easy-to-use tool that should facilitate the use and interpretation of brain atrophy in both research and clinical settings. 


\section{Conflicts of Interest}

M. Battaglini, G. Gentile, L. Luchetti, A. Giorgio have nothing to disclose.

H. Vrenken has received research grants from Pfizer, MerckSerono, Novartis and Teva, and speaker honoraria from Novartis and MerckSerono; all funds were paid directly to his institution.

F.Barkhof serves as a consultant for Bayer Schering Pharma, Sanofi-Aventis, Genzyme, BiogenIdec, Teva, Novartis, Roche, Synthon BV and Jansen Research.

K. S, Cover has nothing to disclose.

R. Bakshi has received consulting fees from EMD Serono, Genentech, Sanofi-Genzyme, and Novartis and research support from Biogen, EMD-Serono, Novartis, and Sanofi-Genzyme

R. Chu has nothing to disclose.

MP Sormani has received personal compensation for consulting services and for speaking activities from Genzyme, Merck Serono, Teva, Synthon, Actelion, Novartis and Biogen Idec.

C Enzinger received funding for traveling and speaker honoraria from Biogen Idec, Bayer Schering Pharma, Merck Serono, Novartis, Genzyme and Teva Pharmaceutical Industries Ltd./sanofi-aventis; received research support from Merck Serono, Biogen Idec, and Teva Pharmaceutical Industries Ltd./sanofi-aventis; and serves on scientific advisory boards for Bayer Schering Pharma, Biogen Idec, Merck Serono, Novartis, Genzyme, Roche, and Teva Pharmaceutical Industries Ltd./sanofiAventis.

S. Ropele has nothing to disclose. 
O Ciccarelli receives research grant support from the Multiple Sclerosis Society of Great Britain and Northern Ireland, the NIHR UCLH Biomedical Research Centre; she is a consultant for Teva, Roche, Novartis, Biogen, Genzyme and GE. She is an Associate Editor for Neurology, for which she receives an honorarium.

C. Wheeler-Kingshott is on the editorial board of Functional Neurology and receives research grants (PI and co-applicant) from ISRT, EPSRC, Wings for Life, UK MS Society, Horizon2020, Biogen and Novartis.

M. Yiannakas has nothing to disclose.

M. Filippi is Editor-in-Chief of the Journal of Neurology; has received compensation for consulting services and/or speaking activities from Biogen Idec, Excemed, Novartis, and Teva Pharmaceutical Industries; and receives research support from Biogen Idec, Teva Pharmaceutical Industries, Novartis, Italian Ministry of Health, Fondazione Italiana Sclerosi Multipla, Cure PSP, Alzheimer's Drug Discovery Foundation (ADDF), the Jacques and Gloria Gossweiler Foundation (Switzerland), and ARiSLA (Fondazione Italiana di Ricerca per la SLA).

M.A. Rocca received speaker's honoraria from Biogen Idec, Novartis, TEVA, Genzyme, Merck Serono and Roche and receives research support from the Italian Ministry of Health and Fondazione Italiana Sclerosi Multipla.

P. Preziosa received speakers honoraria from Biogen Idec, Novartis, Merck Serono and Excemed

A. Gallo and A.Bisecco have nothing to disclose.

J. Palace: consultant for Merck Serono, Biogen Idec, Novartis, Teva, Chugai Pharma, Alexion, and Bayer Schering; research funding from Merck Serono, Novartis, Biogen Idec, and Bayer Schering. 
Y. Kong has nothing to disclose.

D. Horakova was supported by the Czech Ministry of Education project Progres Q27/LF1. She also received compensation for travel, speaker honoraria and consultant fees from Biogen Idec, Novartis, Merck, Bayer, Sanofi Genzyme, Roche, and Teva, as well as support for research activities from Biogen Idec.

M. Vaneckova was supported by Czech Ministry of Health, grant RVO-VFN 64165. She received compensation for speaker honoraria, travel and consultant fees from Biogen Idec, Sanofi Genzyme, Novartis, Merck and Teva, as well as support for research activities from Biogen Idec.

C. Gasperini has received speaker honoraria from Bayer Schering Pharma, Sanofi-Aventis, Merck Serono, Biogen Idec, and Novartis

S. Ruggeri has nothing to disclose.

N De Stefano has received honoraria from Biogen-Idec, Genzyme, Merck Serono, Novartis, Roche and Teva for consulting services, speaking and travel support. He serves on advisory boards for Merck Serono, Novartis, Biogen-Idec, Roche, and Genzyme, he has received research grant support from the Italian MS Society.

\section{Acknowledgements}

We wish to thank Prof. Mark Jenkinson for the several useful talks on this topic. 
Table 1. Demographics of the healthy individuals and MRI acquisition characteristics of each participating centre

\begin{tabular}{|c|c|c|c|c|c|c|c|c|}
\hline Center & $\begin{array}{l}\text { No. of } \\
\text { participants } \\
\text { (male) }\end{array}$ & $\begin{array}{l}\text { Mean } \\
\text { age } \\
\text { (SD) }\end{array}$ & $\begin{array}{l}\text { Magnetic } \\
\text { field strength } \\
\text { and vendor }\end{array}$ & $\begin{array}{l}\text { Slice } \\
\text { thickness } \\
\text { (mm) }\end{array}$ & $\begin{array}{l}\text { Acquisition } \\
\text { plane }\end{array}$ & $\begin{array}{l}\text { T1 Sequence } \\
\text { Types }\end{array}$ & $\begin{array}{l}\text { Head coil } \\
\text { channels }\end{array}$ & $\begin{array}{l}\text { Distortion } \\
\text { correction }\end{array}$ \\
\hline ADNI & $\begin{array}{l}96 \\
(48)\end{array}$ & $\begin{array}{l}76.3 \\
(4.6)\end{array}$ & $1.5 \mathrm{~T} \mathrm{GE}$ & 1.2 & Axial & & & Yes \\
\hline Amsterdam & $\begin{array}{l}56 \\
(24)\end{array}$ & $\begin{array}{l}43.1 \\
(9.8)\end{array}$ & 1.5T Siemens & 1.3 & Coronal & MPRAGE & 8-channels & No \\
\hline Boston & $\begin{array}{l}51 \\
(17)\end{array}$ & $\begin{array}{l}38.7 \\
(9.2)\end{array}$ & $\begin{array}{l}\text { 1.5T GE } \\
\text { 3T GE } \\
\text { 3T Siemens }\end{array}$ & $\begin{array}{l}1.2 \\
1.6 \\
1\end{array}$ & $\begin{array}{l}\text { Sagittal } \\
\text { Coronal } \\
\text { Sagittal }\end{array}$ & 3D-MTEFT & & Yes \\
\hline Graz & $\begin{array}{l}155 \\
(80)\end{array}$ & $\begin{array}{l}29.7 \\
(16)\end{array}$ & 3T Siemens & 1 & Sagittal & MPRAGE & 12 channels & No \\
\hline London & $\begin{array}{l}87 \\
(43)\end{array}$ & $\begin{array}{l}36.7 \\
(11.1)\end{array}$ & 3T Philips & 1 & Sagittal & 3D-TFE & 32-channels & Yes \\
\hline Milan & $\begin{array}{l}157 \\
(76)\end{array}$ & $\begin{array}{l}27.2 \\
(10.8)\end{array}$ & 3T Philips & 0.8 & Axial & $\begin{array}{l}\text { 3D, short TR } \\
\text { spoiled, } \\
\text { Gradient echo }\end{array}$ & 8-channels & Yes \\
\hline Naples & $\begin{array}{l}26 \\
(15)\end{array}$ & $\begin{array}{l}41 \\
(9.9)\end{array}$ & 3T GE & 1.2 & Sagittal & $\begin{array}{l}\text { 3D-T1 } \\
\text { magnetization } \\
\text { prepared Fast } \\
\text { Spoiled } \\
\text { Gradient echo }\end{array}$ & 8-channels & No \\
\hline Oxford & $\begin{array}{l}15 \\
(4)\end{array}$ & $\begin{array}{l}50 \\
(15.9)\end{array}$ & 3T Siemens & 1 & Axial & MPRAGE & 32-channels & No \\
\hline Prague & $\begin{array}{l}83 \\
(38)\end{array}$ & $\begin{array}{l}35.5 \\
(10)\end{array}$ & 1.5T Philips & 1 & Axial & 3D T1 FFE & & No \\
\hline Rome & $\begin{array}{l}13 \\
(7)\end{array}$ & $\begin{array}{l}36.5 \\
(10.5)\end{array}$ & 1.5T Siemens & 1 & Axial & $\begin{array}{l}\text { 3D T1 } \\
\text { Gradient echo }\end{array}$ & 6 channels & No \\
\hline Siena & $\begin{array}{l}37 \\
(17)\end{array}$ & $\begin{array}{l}37.4 \\
(10.2)\end{array}$ & 1.5 T Philips & 3 & Axial & Gradient echo & 8-channels & No \\
\hline Total & 720 & & & & & & & \\
\hline
\end{tabular}


Table 2. Intra-subject variability as assessed in participants with follow-up $<1$ month. Number of participants excluded after quality control and the causes of exclusion are also reported. Intrasubject variability estimations are expressed as mean (range).

\begin{tabular}{|c|c|c|c|c|}
\hline Center & $\begin{array}{l}\text { No. of } \\
\text { participants } \\
\text { with follow-up } \\
\text { <1 month } \\
\end{array}$ & $\begin{array}{l}\text { No. of excluded } \\
\text { participants }\end{array}$ & $\begin{array}{l}\text { Causes of } \\
\text { exclusion }\end{array}$ & $\begin{array}{l}\text { Intra-subject } \\
\text { variability }\end{array}$ \\
\hline ADNI & 96 & 1 & $\begin{array}{l}\text { Partial brain } \\
\text { coverage }\end{array}$ & $0.22 \%(0.001-1.78)$ \\
\hline Amsterdam & 1 & 0 & & $0.65 \%$ \\
\hline Boston & 8 & 0 & & $0.37 \%(0.04-0.72)$ \\
\hline Graz & 68 & 0 & & $0.19 \%(0.003-1.23)$ \\
\hline London & 51 & 8 & $\begin{array}{l}\text { Poor GM/CSF } \\
\text { contrast }\end{array}$ & $0.37 \%(0.003-1.23)$ \\
\hline Milan & 131 & 3 & Motion artifacts & $0.22 \%(0.009-0.92)$ \\
\hline Naples & 6 & 0 & & $0.1 \%(0.02-0.28)$ \\
\hline Oxford & 15 & 0 & & $0.08 \%(0.001-0.24)$ \\
\hline Prague & 3 & 0 & & $0.1 \%(0.02-0.16)$ \\
\hline Rome & 13 & 0 & & $0.19 \%(0.01-1)$ \\
\hline Siena & 0 & 0 & & \\
\hline Total & 392 & $12(3.07 \%)$ & & $0.25 \%(0.0001-1.78)$ \\
\hline
\end{tabular}


Table 3. Normative data as assessed in healthy individuals with follow-up $>6$ months. Number of participants excluded after quality control and the causes of exclusion are also reported

\begin{tabular}{|c|c|c|c|}
\hline Center & $\begin{array}{l}\text { No. of } \\
\text { participants } \\
\text { with follow-up } \\
>6 \text { months }\end{array}$ & $\begin{array}{l}\text { No. of excluded } \\
\text { participants }\end{array}$ & Causes of exclusion \\
\hline ADNI & 78 & 2 & Poor repositioning \\
\hline Amsterdam & 54 & 2 & Motion artifacts \\
\hline Boston & 32 & 2 & $\begin{array}{l}\text { Poor repositioning } \\
\text { Motion artifacts }\end{array}$ \\
\hline Graz & 14 & 5 & $\begin{array}{l}\text { Motion artifacts } \\
\text { Poor repositioning }\end{array}$ \\
\hline London & 57 & 18 & $\begin{array}{l}\text { Poor GM/CSF } \\
\text { contrast }\end{array}$ \\
\hline Milan & 28 & 0 & \\
\hline Naples & 26 & 2 & Motion artifacts \\
\hline Oxford & 15 & 3 & $\begin{array}{l}\text { Motion artifacts } \\
\text { Field inhomogeneities }\end{array}$ \\
\hline Prague & 80 & 3 & $\begin{array}{l}\text { Motion artifacts } \\
\text { Partial brain coverage }\end{array}$ \\
\hline Rome & 0 & 0 & \\
\hline Siena & 37 & 0 & \\
\hline Total & 421 & $37(8.79 \%)$ & \\
\hline
\end{tabular}


Table 4. Predicted rate of $\mathrm{PBVC} / \mathrm{y}$ at $1.5 \mathrm{~T}$ for different age ranges. The $80^{\text {th }}$ and $95^{\text {th }}$ normative percentiles (NP) for 1 and 2 years of follow-up are provided (see text for details).

\begin{tabular}{|c|c|c|c|c|}
\hline & \multicolumn{4}{|c|}{$1.5 T$} \\
\hline $\begin{array}{l}\text { Age range } \\
\text { (years) }\end{array}$ & $\begin{array}{l}\text { Predicted } \\
\text { PBVC/y }\end{array}$ & $\begin{array}{l}\text { Follow-up } \\
\text { length }\end{array}$ & $80^{\text {th }} N P$ & $95^{\text {th }} \mathrm{NP}$ \\
\hline \multirow{2}{*}{$20-30$} & \multirow{2}{*}{-0.17} & 1 year & -0.48 & -0.87 \\
\hline & & 2 years & -0.37 & -0.58 \\
\hline \multirow{2}{*}{$30-40$} & \multirow{2}{*}{-0.23} & 1 year & -0.55 & -0.98 \\
\hline & & 2 years & -0.44 & -0.67 \\
\hline \multirow{2}{*}{$40-50$} & \multirow{2}{*}{-0.29} & 1 year & -0.64 & -1.10 \\
\hline & & 2 years & -0.51 & -0.76 \\
\hline \multirow{2}{*}{$50-60$} & \multirow{2}{*}{-0.36} & 1 year & -0.72 & -1.24 \\
\hline & & 2 years & -0.59 & -0.86 \\
\hline \multirow{2}{*}{$60-70$} & \multirow{2}{*}{-0.43} & 1 year & -0.82 & -1.41 \\
\hline & & 2 years & -0.67 & -0.96 \\
\hline \multirow{2}{*}{$70-80$} & \multirow{2}{*}{-0.50} & 1 year & -0.92 & -1.60 \\
\hline & & 2 years & -0.76 & -1.09 \\
\hline
\end{tabular}


Table 5. Predicted rate of PBVC/y at $3 \mathrm{~T}$ for different age ranges. The $80^{\text {th }}$ and $95^{\text {th }}$ normative percentiles (NP) for 1 and 2 years of follow-up are provided (see text for details).

\begin{tabular}{|c|c|c|c|c|}
\hline \multicolumn{5}{|c|}{$3 T$} \\
\hline $\begin{array}{l}\text { Age range } \\
\text { (years) }\end{array}$ & $\begin{array}{l}\text { Predicted } \\
P B V C / y\end{array}$ & $\begin{array}{l}\text { Follow-up } \\
\text { length }\end{array}$ & $80^{\text {th }} \mathrm{NP}$ & $95^{\text {th }} \mathrm{NP}$ \\
\hline \multirow{2}{*}{$20-30$} & \multirow{2}{*}{-0.05} & 1 year & -0.34 & -0.68 \\
\hline & & 2 years & -0.24 & -0.44 \\
\hline \multirow{2}{*}{$30-40$} & \multirow{2}{*}{-0.11} & 1 year & -0.41 & -0.77 \\
\hline & & 2 years & -0.30 & -0.51 \\
\hline \multirow{2}{*}{$40-50$} & \multirow{2}{*}{-0.17} & 1 year & -0.48 & -0.87 \\
\hline & & 2 years & -0.37 & -0.59 \\
\hline \multirow{2}{*}{$50-60$} & \multirow{2}{*}{-0.23} & 1 year & -0.56 & -0.99 \\
\hline & & 2 years & -0.44 & -0.67 \\
\hline \multirow{2}{*}{$60-70$} & \multirow{2}{*}{-0.30} & 1 year & -0.64 & -1.11 \\
\hline & & 2 years & -0.51 & -0.76 \\
\hline \multirow{2}{*}{$70-80$} & \multirow{2}{*}{-0.36} & 1 year & -0.73 & -1.25 \\
\hline & & 2 years & -0.59 & -0.86 \\
\hline
\end{tabular}




\section{Figure legend}

Figure 1. Scatterplot of PBVC/y (Y-axis) against age (X-axis) of the 789 pairs of MRI images analysed with SIENA.

Figure 2. Normative values of PBVC/y as obtained with SIENA at $1.5 \mathrm{~T}$ (left side) and $3 \mathrm{~T}$ (right side) for 1-year (top) and 2-year (bottom) follow-up. Predicted values as resulted from the model are shown in each panel (blu line). Also note, in each panel, the values of PBVC/y within the $80^{\text {th }}$ and $95^{\text {th }}$ normative percentiles (NP, light blu areas) and higher than the $95^{\text {th }} \mathrm{NP}$ (pink areas). 


\section{References}

Ashburner J, Ridgway GR. Symmetric diffeomorphic modeling of longitudinal structural MRI. Front Neurosci. 2013;6:197.

Battaglini M, Jenkinson M, De Stefano N. SIENA-XL for improving the assessment of gray and white matter volume changes on brain MRI. Human brain mapping. 2018 Mar;39(3):1063-77.

Biberacher V, Schmidt P, Keshavan A, Boucard CC, Righart R, Samann P, et al. Intra- and interscanner variability of magnetic resonance imaging based volumetry in multiple sclerosis. NeuroImage. 2016 Nov 15;142:188-97.

Chu R, Hurwitz S, Tauhid S, Bakshi R. Automated segmentation of cerebral deep gray matter from MRI scans: effect of field strength on sensitivity and reliability. BMC Neurol. 2017 Sep $5 ; 17(1): 172$.

Chu R, Tauhid S, Glanz BI, Healy BC, Kim G, Oommen VV, et al. Whole Brain Volume Measured from 1.5T versus 3T MRI in Healthy Participants and Patients with Multiple Sclerosis. J Neuroimaging. 2016 Jan-Feb;26(1):62-7.

Cover KS, van Schijndel RA, Popescu V, van Dijk BW, Redolfi A, Knol DL, et al. The SIENA/FSL whole brain atrophy algorithm is no more reproducible at $3 \mathrm{~T}$ than $1.5 \mathrm{~T}$ for Alzheimer's disease. Psychiatry research. 2014 Oct 30;224(1):14-21.

De Stefano N, Airas L, Grigoriadis N, Mattle HP, O'Riordan J, Oreja-Guevara C, et al. Clinical relevance of brain volume measures in multiple sclerosis. CNS Drugs. 2014 Feb;28(2):147-56.

De Stefano N, Silva DG, Barnett MH. Effect of Fingolimod on Brain Volume Loss in Patients with Multiple Sclerosis. CNS Drugs. 2017 Apr;31(4):289-305.

De Stefano N, Stromillo ML, Giorgio A, Bartolozzi ML, Battaglini M, Baldini M, et al. Establishing pathological cut-offs of brain atrophy rates in multiple sclerosis. Journal of neurology, neurosurgery, and psychiatry. 2016 Jan;87(1):93-9.

Driscoll I, Davatzikos C, An Y, Wu X, Shen D, Kraut M, et al. Longitudinal pattern of regional brain volume change differentiates normal aging from MCI. Neurology. 2009 Jun 2;72(22):190613.

Enzinger C, Fazekas F, Matthews PM, Ropele S, Schmidt H, Smith S, et al. Risk factors for progression of brain atrophy in aging: six-year follow-up of normal participants. Neurology. 2005 May 24;64(10):1704-11.

Fotenos AF, Snyder AZ, Girton LE, Morris JC, Buckner RL. Normative estimates of crosssectional and longitudinal brain volume decline in aging and AD. Neurology. 2005 Mar 22;64(6):1032-9.

Frisoni GB, Fox NC, Jack CR, Jr., Scheltens P, Thompson PM. The clinical use of structural MRI in Alzheimer disease. Nat Rev Neurol. 2010 Feb;6(2):67-77.

Giorgio A, De Stefano N. Clinical use of brain volumetry. J Magn Reson Imaging. 2013 Jan;37(1):1-14.

Guevara C, Bulatova K, Soruco W, Gonzalez G, Farias GA. Retrospective Diagnosis of Parkinsonian Syndromes Using Whole-Brain Atrophy Rates. Front Aging Neurosci. 2017;9:99.

Hedman AM, van Haren NE, Schnack HG, Kahn RS, Hulshoff Pol HE. Human brain changes across the life span: a review of 56 longitudinal magnetic resonance imaging studies. Human brain mapping. 2012 Aug;33(8):1987-2002.

Hotter A, Esterhammer R, Schocke MF, Seppi K. Potential of advanced MR imaging techniques in the differential diagnosis of parkinsonism. Mov Disord. 2009;24 Suppl 2:S711-20.

Jenkinson M, Beckmann CF, Behrens TE, Woolrich MW, Smith SM. Fsl. NeuroImage. 2012 Aug 15;62(2):782-90.

Kruggel F, Turner J, Muftuler LT. Impact of scanner hardware and imaging protocol on image quality and compartment volume precision in the ADNI cohort. NeuroImage. 2010 Feb $1 ; 49(3): 2123-33$. 
Liu RS, Lemieux L, Bell GS, Sisodiya SM, Shorvon SD, Sander JW, et al. A longitudinal study of brain morphometrics using quantitative magnetic resonance imaging and difference image analysis. NeuroImage. 2003 Sep;20(1):22-33.

Mak E, Su L, Williams GB, Watson R, Firbank M, Blamire AM, et al. Longitudinal assessment of global and regional atrophy rates in Alzheimer's disease and dementia with Lewy bodies. Neuroimage Clin. 2015;7:456-62.

Marcus DS, Fotenos AF, Csernansky JG, Morris JC, Buckner RL. Open access series of imaging studies: longitudinal MRI data in nondemented and demented older adults. Journal of cognitive neuroscience. 2010 Dec;22(12):2677-84.

Nakamura K, Guizard N, Fonov VS, Narayanan S, Collins DL, Arnold DL. Jacobian integration method increases the statistical power to measure gray matter atrophy in multiple sclerosis. Neuroimage Clin. 2013;4:10-7.

Opfer R, Ostwaldt AC, Sormani MP, Gocke C, Walker-Egger C, Manogaran P, et al. Estimates of age-dependent cutoffs for pathological brain volume loss using SIENA/FSL-a longitudinal brain volumetry study in healthy adults. Neurobiology of aging. 2017 May;65:1-6.

Opfer R, Ostwaldt AC, Walker-Egger C, Manogaran P, Sormani MP, De Stefano N, et al. Withinpatient fluctuation of brain volume estimates from short-term repeated MRI measurements using SIENA/FSL. Journal of neurology. 2018 May;265(5):1158-65.

Popescu V, Battaglini M, Hoogstrate WS, Verfaillie SC, Sluimer IC, van Schijndel RA, et al. Optimizing parameter choice for FSL-Brain Extraction Tool (BET) on 3D T1 images in multiple sclerosis. NeuroImage. 2012 Jul 16;61(4):1484-94.

Resnick SM, Pham DL, Kraut MA, Zonderman AB, Davatzikos C. Longitudinal magnetic resonance imaging studies of older adults: a shrinking brain. J Neurosci. 2003 Apr 15;23(8):3295301.

Reuter M, Fischl B. Avoiding asymmetry-induced bias in longitudinal image processing. NeuroImage. 2011 Jul 1;57(1):19-21.

Rocca MA, Battaglini M, Benedict RH, De Stefano N, Geurts JJ, Henry RG, et al. Brain MRI atrophy quantification in MS: From methods to clinical application. Neurology. 2017 Jan 24;88(4):403-13.

Sastre-Garriga J, Pareto D, Rovira A. Brain Atrophy in Multiple Sclerosis: Clinical Relevance and Technical Aspects. Neuroimaging Clin N Am. 2017 May;27(2):289-300.

Sastre-Garriga J, Tur C, Pareto D, Vidal-Jordana A, Auger C, Rio J, et al. Brain atrophy in natalizumab-treated patients: A 3-year follow-up. Multiple sclerosis (Houndmills, Basingstoke, England). 2015 May;21(6):749-56.

Smith SM. Fast robust automated brain extraction. Human brain mapping. 2002 Nov;17(3):143-55.

Smith SM, Zhang Y, Jenkinson M, Chen J, Matthews PM, Federico A, et al. Accurate, robust, and automated longitudinal and cross-sectional brain change analysis. NeuroImage. 2002 Sep;17(1):479-89.

Takao H, Hayashi N, Ohtomo K. A longitudinal study of brain volume changes in normal aging. Eur J Radiol. 2012 Oct;81(10):2801-4.

Tustison NJ, Avants BB, Cook PA, Zheng Y, Egan A, Yushkevich PA, et al. N4ITK: improved N3 bias correction. IEEE Trans Med Imaging. 2010 Jun;29(6):1310-20.

van Maurik IS, Zwan MD, Tijms BM, Bouwman FH, Teunissen CE, Scheltens P, et al. Interpreting Biomarker Results in Individual Patients With Mild Cognitive Impairment in the Alzheimer's Biomarkers in Daily Practice (ABIDE) Project. JAMA Neurol. 2017 Dec 1;74(12):1481-91.

Vrenken H, Jenkinson M, Horsfield MA, Battaglini M, van Schijndel RA, Rostrup E, et al. Recommendations to improve imaging and analysis of brain lesion load and atrophy in longitudinal studies of multiple sclerosis. Journal of neurology. 2013 Oct;260(10):2458-71. 